\title{
On Set-Valued Complementarity Problems
}

\author{
Jinchuan Zhou, ${ }^{1}$ Jein-Shan Chen, ${ }^{2}$ and Gue Myung Lee ${ }^{3}$ \\ ${ }^{1}$ Department of Mathematics, School of Science, Shandong University of Technology, Zibo 255049, China \\ ${ }^{2}$ Department of Mathematics, National Taiwan Normal University, Taipei 11677, Taiwan \\ ${ }^{3}$ Department of Applied Mathematics, Pukyong National University, Busan 608-737, Republic of Korea
}

Correspondence should be addressed to Jein-Shan Chen; jschen@math.ntnu.edu.tw

Received 18 September 2012; Accepted 14 December 2012

Academic Editor: Mohamed Tawhid

Copyright (C) 2013 Jinchuan Zhou et al. This is an open access article distributed under the Creative Commons Attribution License, which permits unrestricted use, distribution, and reproduction in any medium, provided the original work is properly cited.

\begin{abstract}
This paper investigates the set-valued complementarity problems (SVCP) which poses rather different features from those that classical complementarity problems hold, due to the fact that he index set is not fixed, but dependent on $x$. While comparing the set-valued complementarity problems with the classical complementarity problems, we analyze the solution set of SVCP. Moreover, properties of merit functions for SVCP are studied, such being as level bounded and error bounded. Finally, some possible research directions are discussed.
\end{abstract}

\section{Motivations and Preliminaries}

The set-valued complementarity problem (SVCP) is to find $x \in$ $\mathbb{R}^{n}$ such that

$$
x \geq 0, \quad y \geq 0, \quad x^{T} y=0, \quad \text { for some } y \in \Theta(x),
$$

where $\Theta: \mathbb{R}_{+}^{n} \rightrightarrows \mathbb{R}^{n}$ is a set-valued mapping. The setvalued complementarity problem plays an important role in the sensitivity analysis of complementarity problems [1] and economic equilibrium problems [2]. However, there has been very little study on the set-valued complementarity problems compared to the classical complementarity problems. In fact, the SVCP (1) can be recast as follows, which is denoted by $\operatorname{SVNCP}(F, \Omega)$ to find $x \in \mathbb{R}^{n}$, such that

$$
x \geq 0, \quad F(x, w) \geq 0, \quad x^{T} F(x, w)=0,
$$

for some $w \in \Omega(x)$,

where $F: \mathbb{R}^{n} \times \mathbb{R}^{m} \rightarrow \mathbb{R}^{n}$ and $\Omega: \mathbb{R}^{n} \rightrightarrows \mathbb{R}^{m}$ are a set-valued mapping. To see this, if letting

$$
\Theta(x)=\bigcup_{w \in \Omega(x)}\{F(x, w)\}
$$

then (1) reduces to (2). Conversely, if $F(x, w)=w$ and $\Omega(x)=$ $\Theta(x)$, then (2) takes the form of (1).
The $\operatorname{SVNCP}(F, \Omega)$ given as in (2) provides an unified framework for several interesting and important problems in optimization fields described as below.

(i) Nonlinear complementarity problem [1], which is to find $x \in \mathbb{R}^{n}$, such that

$$
x \geq 0, \quad F(x) \geq 0, \quad\langle x, F(x)\rangle=0 .
$$

This corresponds to $F(x, w):=F(x)+w$ and $\Omega(x)=$ $\{0\}$ for all $x \in \mathbb{R}^{n}$. In other words, the set-valued complementarity problem reduces to the classical complementarity problem under such case.

(ii) Extended linear complementarity problem [3, 4], which is to find $x, w \in \mathbb{R}^{n}$, such that

$$
\begin{array}{r}
x \geq 0, \quad w \geq 0, \quad x^{T} w=0, \\
\text { with } M_{1} x-M_{2} w \in P,
\end{array}
$$

where $M_{1}, M_{2} \in \mathbb{R}^{m \times n}$ and $P \subseteq \mathbb{R}^{m}$ are a poly-hedron. This corresponds to $F(x, w)=w$ and $\Omega(x)=\left\{w \mid M_{1} x-\right.$ $\left.M_{2} w \in P\right\}$. In particular, when $P=\{q\}$, it further reduces to the horizontal linear complementarity problem and to the usual linear complementarity problem, in addition to $M_{2}$ being an identify matrix. 
(iii) Implicit complementarity problem [5], which is to find $x, w \in \mathbb{R}^{n}$ and $z \in \mathbb{R}^{m}$, such that

$x \geq 0, \quad w \geq 0, \quad x^{T} w=0, \quad$ with $F(x, w, z)=0$,

where $F: \mathbb{R}^{2 n \times m} \rightarrow \mathbb{R}^{l}$. This can be rewritten as

$$
x \geq 0, \quad w \geq 0, \quad x^{T} w=0,
$$

with $w$ satisfying $F(x, w, z)=0$ for some $z$.

This is clearly an $\operatorname{SVNCP}(F, \Omega)$ where $F(x, w)=w$ and $\Omega(x)=$ $\cup_{z \in \mathbb{R}^{m}}\{w \mid F(x, w, z)=0\}$.

(iv) Mixed nonlinear complementarity problem, which is to find $x \in \mathbb{R}^{n}$, and $w \in \mathbb{R}^{m}$ such that

$$
\begin{array}{r}
x \geq 0, \quad F(x, w) \geq 0, \quad\langle x, F(x, w)\rangle=0, \\
\text { with } G(x, w)=0 .
\end{array}
$$

This is an $\operatorname{SVNCP}(F, \Omega)$ where it corresponds to $\Omega(x)=\{x \mid$ $G(x, w)=0\}$. Note that the mixed nonlinear complementarity problem is a natural extension of Karush-Kuhn-Tucker (KKT) conditions for the following nonlinear programming:

$$
\begin{array}{ll}
\min & f(x) \\
\text { s.t. } & g_{i}(x) \leq 0, \quad i=1,2, \ldots, m, \\
h_{j}(x)=0, \quad j=1, \ldots, l .
\end{array}
$$

To see this, we first write out the KKT conditions as follows:

$$
\begin{gathered}
\nabla f(x)+\sum_{i=1}^{m} \lambda_{i} \nabla g_{i}(x)+\sum_{j=1}^{l} \mu_{j} \nabla h_{j}(x)=0, \\
h(x)=0, \\
g(x) \leq 0, \quad \lambda \geq 0, \quad\langle\lambda, g(x)\rangle=0,
\end{gathered}
$$

where $g(x):=\left(g_{1}(x), \ldots, g_{m}(x)\right), h(x):=\left(h_{1}(x), \ldots, h_{l}(x)\right)$, and $\lambda:=\left(\lambda_{1}, \ldots, \lambda_{m}\right)$. Then, letting $w:=(\lambda, \mu), F(x, w):=$ $-g(x)$, and

$$
G(x, w):=\left(\begin{array}{c}
\nabla f(x)+\sum_{i=1}^{m} \lambda_{i} \nabla g_{i}(x)+\sum_{j=1}^{l} \mu \nabla h_{j}(x) \\
h(x)
\end{array}\right)
$$

implies that the KKT system (10) becomes a mixed complementarity problem.

Besides the above various complementarity problems, $\operatorname{SVNCP}(F, \Omega)$ has a close relation with the Quasi-variational inequality, a special of the extended general variational inequalities $[6,7]$, and min-max programming, which is elaborated as below.

(v) Quasi-variational inequality [2]. Given a point-topoint map $F$ from $\mathbb{R}^{n}$ to itself and a point-to-set map $K$ from $\mathbb{R}^{n}$ into subsets of $\mathbb{R}^{n}$, the Quasi-variational inequality $\mathrm{QVI}(K, F)$ is to find a vector $x \in K(x)$, such that

$$
\langle F(x), y-x\rangle \geq 0, \quad \forall y \in K(x) .
$$

It is well-known that $\mathrm{QVI}(K, F)$ reduces to the classical nonlinear complementarity problem when $K(x)$ is independent of $x$, say, $K(x)=\mathbb{R}_{+}^{n}$ for all $x$. Now, let us explain why it is related to $\operatorname{SVNCP}(F, \Omega)$. To this end, given $x \in \mathbb{R}^{n}$, we define $I(x)=\left\{i \mid F_{i}(x)>0\right\}$, and let

$$
\begin{array}{r}
K(x)=\left\{x \mid x_{i} \geq 0 \text { for } i \in I \backslash I(x)\right. \\
\text { and } \left.x_{i}=0 \text { for } i \in I(x)\right\} .
\end{array}
$$

Clearly, $0 \in K(x)$ which says $\langle x, F(x)\rangle \leq 0$ by taking $y=0$ in (12). Note that $x \geq 0$ because $x \in K(x)$. Next, we will claim that $F_{i}(x) \geq 0$ for all $i=1,2, \ldots, n$. It is enough to consider the case where $i \in I \backslash I(x)$. Under such case, by taking $y=\beta e_{i}$ in (12) with $\beta$ being an arbitrarily positive scalar, we have $\beta F_{i}(x) \geq F(x)^{T} x$. Since $\beta$ can be made sufficiently large, it implies that $F_{i}(x) \geq 0$. Thus, we obtain $F(x)^{T} x \geq 0$. In summary, under such case, $\mathrm{QVI}(K, F)$ becomes

$$
x \geq 0, \quad F(x) \geq 0, \quad x^{T} F(x)=0, \quad \text { with } x \in K(x)
$$

which is an $\operatorname{SVNCP}(F, \Omega)$.

(vi) Min-max programming [8], which is to solve the following problem:

$$
\min _{x \in \mathbb{R}_{+}^{n}} \max _{w \in \Omega} f(x, w)
$$

where $f: \mathbb{R}^{n} \times \Omega \rightarrow \mathbb{R}$ is a continuously differentiable function, and $\Omega$ is a compact subset in $\mathbb{R}^{m}$. First, we define $\psi(x):=\max _{w \in \Omega} f(x, w)$. Although $\psi$ is not necessarily Frechet differentiable, it is directional differentiable (even semi-smooth), see [9]. Now, let us check the first-order necessary conditions for problem (15). In fact, if $x^{*}$ is a local minimizer of (15), then

$$
\begin{array}{r}
\psi^{\prime}\left(x^{*} ; x-x^{*}\right)=\max _{w \in \Omega\left(x^{*}\right)}\left\langle\nabla_{x} f\left(x^{*}, w\right), x-x^{*}\right\rangle \geq 0, \\
\forall x \in \mathbb{R}_{+}^{n},
\end{array}
$$

which is equivalent to

$$
\inf _{x \in \mathbb{R}_{+}^{n}} \max _{w \in \Omega\left(x^{*}\right)}\left\langle\nabla_{x} f\left(x^{*}, w\right), x-x^{*}\right\rangle=0,
$$

where $\Omega(x)$ means the active set at $x$, that is, $\Omega(x):=\{w \in$ $\Omega \mid \psi(x)=f(x, w)\}$. At our first glance, the formula (17) is not related to $\operatorname{SVNCP}(F, \Omega)$. Nonetheless, we will show that if $\Omega$ is convex and the function $f(x, \cdot)$ is concave over $\Omega$, then the first-order necessary conditions form an $\operatorname{SVNCP}(F, \Omega)$, see below proposition.

Proposition 1. Let $\Omega$ be nonempty, compact, and convex set in $\mathbb{R}^{m}$. Suppose that, for each $x$, the function $f(x, \cdot)$ is concave over $\Omega$. If $x^{*}$ is a local optimal solution of (15), then there exists $w^{*} \in \Omega\left(x^{*}\right)$, such that

$$
x^{*} \geq 0, \quad \nabla_{x} f\left(x^{*}, w^{*}\right) \geq 0, \quad\left\langle\nabla_{x} f\left(x^{*}, w^{*}\right), x^{*}\right\rangle=0 .
$$


Proof. Note first that for each $x$ the inner problem

$$
\psi(x):=\max _{w \in \Omega} f(x, w)
$$

is a concave optimization problem, since $f(x, \cdot)$ is concave and $\Omega$ is convex. This ensures that $\Omega(x)$, which denotes the optimal solution set of (19), is convex as well. Now we claim that the function

$$
h(w):=\left\langle\nabla_{x} f\left(x^{*}, w\right), x-x^{*}\right\rangle
$$

is concave over $\Omega\left(x^{*}\right)$. Indeed, for $w_{1}, w_{2} \in \Omega\left(x^{*}\right)$ and $\alpha \in$ $[0,1]$, we have

$$
\begin{aligned}
& h\left(\alpha w_{1}+(1-\alpha) w_{2}\right) \\
& =\left\langle\nabla_{x} f\left(x^{*}, \alpha w_{1}+(1-\alpha) w_{2}\right), x-x^{*}\right\rangle \\
& =\lim _{t \downarrow 0}\left(f\left(x^{*}+t\left(x-x^{*}\right), \alpha w_{1}+(1-\alpha) w_{2}\right)\right. \\
& \left.\quad-f\left(x^{*}, \alpha w_{1}+(1-\alpha) w_{2}\right)\right)(t)^{-1} \\
& =\lim _{t \downarrow 0} \frac{f\left(x^{*}+t\left(x-x^{*}\right), \alpha w_{1}+(1-\alpha) w_{2}\right)-\psi\left(x^{*}\right)}{t} \\
& \geq \lim _{t \downarrow 0}\left(\alpha f\left(x^{*}+t\left(x-x^{*}\right), w_{1}\right)+(1-\alpha)\right. \\
& \left.\quad \times f\left(x^{*}+t\left(x-x^{*}\right), w_{2}\right)-\psi\left(x^{*}\right)\right)(t)^{-1} \\
& =\lim _{t \downarrow 0} \frac{\alpha\left[f\left(x^{*}+t\left(x-x^{*}\right), w_{1}\right)-f\left(x^{*}, w_{1}\right)\right]}{t} \\
& \quad+\lim _{t \downarrow 0} \frac{(1-\alpha)\left[f\left(x^{*}+t\left(x-x^{*}\right), w_{2}\right)-f\left(x^{*}, w_{2}\right)\right]}{t} \\
& =\alpha\left\langle\nabla_{x} f\left(x^{*}, w_{1}\right), x-x^{*}\right\rangle+(1-\alpha) \\
& \quad \times\left\langle\nabla_{x} f\left(x^{*}, w_{2}\right), x-x^{*}\right\rangle \\
& =\alpha h\left(w_{1}\right)+(1-\alpha) h\left(w_{2}\right),
\end{aligned}
$$

where we use the fact that $\alpha w_{1}+(1-\alpha) w_{2} \in \Omega\left(x^{*}\right)$ (since $\Omega\left(x^{*}\right)$ is convex) and $f\left(x^{*}, w\right)=\psi\left(x^{*}\right)$ for all $w \in \Omega\left(x^{*}\right)$. On the other hand, applying the Min-Max Theorem [10, Corollary 37.3.2] to (17) yields

$$
\max _{w \in \Omega\left(x^{*}\right)} \inf _{x \in \mathbb{R}_{+}^{n}}\left\langle\nabla_{x} f\left(x^{*}, w\right), x-x^{*}\right\rangle=0 .
$$

Hence, for arbitrary $\varepsilon>0$, we can find $w_{\varepsilon} \in \Omega\left(x^{*}\right)$, such that

$$
\inf _{x \in \mathbb{R}_{+}^{n}}\left\langle\nabla_{x} f\left(x^{*}, w_{\varepsilon}\right), x-x^{*}\right\rangle \geq-\varepsilon
$$

that is,

$$
\left\langle\nabla_{x} f\left(x^{*}, w_{\varepsilon}\right), x-x^{*}\right\rangle \geq-\varepsilon, \quad \forall x \in \mathbb{R}_{+}^{n} .
$$

In particular, plugging in $x=0$ in (24) implies

$$
\left\langle\nabla_{x} f\left(x^{*}, w_{\varepsilon}\right), x^{*}\right\rangle \leq \varepsilon .
$$

Since $\Omega$ is bounded and $\Omega\left(x^{*}\right)$ is closed, we can assume, without loss of generality, that $w_{\varepsilon} \rightarrow w^{*} \in \Omega\left(x^{*}\right)$ as $\varepsilon \rightarrow 0$. Thus, taking the limit in (25) gives

$$
\left\langle\nabla_{x} f\left(x^{*}, w^{*}\right), x^{*}\right\rangle \leq 0 .
$$

Now, let $x=x^{*}+k e_{i} \in \mathbb{R}_{+}^{n}$. It follows from (24) that

$$
\left(\nabla_{x} f\left(x^{*}, w_{\varepsilon}\right)\right)_{i} \geq-\frac{\varepsilon}{k}
$$

which implies that $\left(\nabla_{x} f\left(x^{*}, w_{\varepsilon}\right)\right)_{i} \geq 0$ by letting $k \rightarrow \infty$, and hence $\left(\nabla_{x} f\left(x^{*}, w^{*}\right)\right)_{i} \geq 0$ for all $i=1,2, \ldots, n$, that is, $\nabla_{x} f\left(x^{*}, w^{*}\right) \geq 0$. This together with (26) means that $\left\langle\nabla_{x} f\left(x^{*}, w^{*}\right), x^{*}\right\rangle=0$. Thus, (18) holds.

From all the above, we have seen that $\operatorname{SVNCP}(F, \Omega)$ given as in (2) covers a range of optimization problems. Therefore, in this paper, we mainly focus on $\operatorname{SVNCP}(F, \Omega)$. Due to its equivalence to SVCP (1), our analysis and results for $\operatorname{SVNCP}(F, \Omega)$ can be carried over to SVCP (1). This paper is organized as follows. In Section 1, connection between $\operatorname{SVNCP}(F, \Omega)$ and various optimization problems is introduced. We recall some background materials in Section 2. Besides comparing the set-valued complementarity problems with the classical complementarity problems, we analyze the solution set of SVCP in Section 3. Moreover, properties of merit functions for SVCP are studied in Section 4, such as level bounded and error bound. Finally, some possible research directions are discussed.

A few words about the notations used throughout the paper. For any $x, y \in \mathbb{R}^{n}$, the inner product is denoted by $x^{T} y$ or $\langle x, y\rangle$. We write $x \geq y$ (or $x>y$ ) if $x_{i} \geq y_{i}$ (or $x_{i}>y_{i}$ ) for all $i=1,2, \ldots, n$. Let $e$ be the vector with all components being 1 , and let $e_{i}$ be the $i$-row of identity matrix. Denote $N_{\infty}:=\cup_{n=1}^{\infty}\{\{n, n+1, \ldots\}\}$. While $\operatorname{SVNCP}(F, \Omega)$ meaning the set-valued nonlinear complementary problem (2), $\operatorname{SVLCP}(M, q, \Omega)$ denotes the linear case, that is, $F(x, w)=$ $M(w) x+q(w)$, where $M: \mathbb{R}^{m} \rightarrow \mathbb{R}^{n \times n}$ and $q: \mathbb{R}^{m} \rightarrow \mathbb{R}^{n}$. For a continuously differentiable function $F: \mathbb{R}^{n} \times \mathbb{R}^{m} \rightarrow$ $\mathbb{R}^{l}$, we denote the $l \times n$ Jacobian matrix of partial derivatives of $F$ at $(\bar{x}, \bar{w})$ with respect to $x$ by $J_{x} F(\bar{x}, \bar{w})$, whereas the transposed Jacobian is denoted by $\nabla_{x} F(\bar{x}, \bar{w})$. For a mapping $H: \mathbb{R}^{n} \rightarrow \mathbb{R}^{m}$, define

$$
\liminf _{x \rightarrow \bar{x}} H(x):=\left(\begin{array}{c}
\liminf _{x \rightarrow \bar{x}} H_{1}(x) \\
\liminf _{x \rightarrow \bar{x}} H_{2}(x) \\
\vdots \\
\liminf _{x \rightarrow \bar{x}} H_{m}(x)
\end{array}\right) .
$$

Given a set-valued mapping $M: \mathbb{R}^{n} \rightrightarrows \mathbb{R}^{m}$, define

$$
\begin{aligned}
& \limsup _{x \rightarrow \bar{x}} M(x) \\
& \quad:=\left\{u \mid \exists x^{n} \longrightarrow \bar{x}, \exists u^{n} \longrightarrow u \text { with } u^{n} \in M\left(x^{n}\right)\right\}, \\
& \liminf _{x \rightarrow \bar{x}} M(x) \\
& \quad:=\left\{u \forall x^{n} \longrightarrow \bar{x}, \exists u^{n} \longrightarrow u \text { with } u^{n} \in M\left(x^{n}\right)\right\} .
\end{aligned}
$$


We say that $M$ is outer semicontinuous at $\bar{x}$, if

$$
\limsup _{x \rightarrow \bar{x}} M(x) \subset M(\bar{x}),
$$

and inner semicontinuous at $\bar{x}$ if

$$
\liminf _{x \rightarrow \bar{x}} M(x) \supset M(\bar{x}) .
$$

We say that $M$ is continuous at $\bar{x}$ if it is both outer semicontinuous and inner semicontinuous at $\bar{x}$. For more details about these functions, please refer to $[9,11]$. Throughout this paper, we always assume that the set-valued mapping $\Omega: \mathbb{R}^{n} \rightrightarrows \mathbb{R}^{m}$ is closed valued; that is, $\Omega(x)$ is closed for all $x \in \mathbb{R}^{n}[11$, Chapter 1].

\section{Focus on $\operatorname{SVLCP}(M, q, \Omega)$}

It is well known that various matrix classes paly different roles in the theory of linear complementarity problem, such as $P$ matrix, $S$-matrix, $Q$-matrix, and $Z$-matrix, see $[1,12]$ for more details. Here we recall some of them which will be needed in the subsequent analysis.

Definition 2. A matrix $M \in \mathbb{R}^{n \times n}$ is said to be an $S$-matrix if there exists $x \in \mathbb{R}^{n}$, such that

$$
x>0, \quad M x>0 .
$$

Note that $M \in \mathbb{R}^{n \times n}$ is an $S$-matrix, if and only if the classical linear complementarity problem $\operatorname{LCP}(M, q)$ is feasible for all $q \in \mathbb{R}^{n}$, see [12, Prop. 3.1.5]. Moreover, the above condition in Definition 2 is equivalent to

$$
x \geq 0, \quad M x>0,
$$

see [13, Remark 2.2]. However, such equivalence fails to hold for its corresponding cases in set-valued complementarity problem. In other words,

$$
x>0, \quad M(w) x>0, \quad \text { for some } w \in \Omega(x)
$$

is not equivalent to

$$
x \geq 0, \quad M(w) x>0, \quad \text { for some } w \in \Omega(x) .
$$

It is clear that (34) implies (35). But, the converse implication does not hold, which is illustrated in Example 3.

Example 3. Let

$$
M(w)=\left(\begin{array}{cc}
w & 0 \\
0 & w
\end{array}\right), \quad \Omega(x)= \begin{cases}\{0,1\}, & x=(1,0) \in \mathbb{R}^{2} ; \\
\{0\}, & \text { otherwise. }\end{cases}
$$

If $M(w) x>0$, then $w=1$, and such case holds only when $x=(1,0)$. Therefore, $(35)$ is satisfied, but (34) is not.

We point out that the set-valued mapping $\Omega(x)$ in Example 3 is indeed outer semi-continuous. A natural question arises: what happens if $\Omega(x)$ is inner semi-continuous? The answer is given in Theorem 4 as below.
Theorem 4. If $\Omega(x)$ is inner semicontinuous, and $M(w)$ is continuous, then (34) and (35) are equivalent.

Proof. We only need to show $(35) \Rightarrow(34)$. Let $H(x)=$ $\max _{w \in \Omega(x)} M(w) x$, and denote by $a_{i}(x)$ the $i$ th row of $M(w)$. Hence $H_{i}(x)=\max _{w \in \Omega(x)} a_{i}(x)^{T} x$. With this, suppose $x_{0}$ is an arbitrary but fixed point, we know that for any $\varepsilon>0$, there exists $w_{0} \in \Omega\left(x_{0}\right)$ such that $a_{i}\left(w_{0}\right)^{T} x_{0}>H_{i}\left(x_{0}\right)-\varepsilon$. Since $\Omega(x)$ is inner semi-continuous, for any $x_{n} \rightarrow x_{0}$, there exists $w_{n} \in \Omega\left(x_{n}\right)$ satisfying $w_{n} \rightarrow w_{0}$. This implies

$$
H_{i}\left(x_{n}\right)=\max _{w \in \Omega\left(x_{n}\right)} a_{i}(w)^{T} x_{n} \geq a_{i}\left(w_{n}\right)^{T} x_{n} .
$$

Then, taking the lower limit yields

$$
\liminf _{n \rightarrow \infty} H_{i}\left(x_{n}\right) \geq \lim _{n \rightarrow \infty} a_{i}\left(w_{n}\right)^{T} x_{n}=a_{i}\left(w_{0}\right)^{T} x_{0}>H_{i}\left(x_{0}\right)-\varepsilon,
$$

where the equality follows from the continuity of $a_{i}(w)$, which is ensured by the continuity of $M(w)$. Because $\varepsilon>0$ is arbitrary, and $\left\{x_{n}\right\}$ is an arbitrary sequence converging to $x_{0}$, we obtain

$$
\liminf _{x \rightarrow x_{0}} H_{i}(x) \geq H_{i}\left(x_{0}\right)
$$

which says $H_{i}$ is lower semi-continuous. This further implies

$$
\begin{aligned}
\liminf _{x \rightarrow x_{0}} H(x) & =\left(\begin{array}{c}
\liminf _{x \rightarrow x_{0}} H_{1}(x) \\
\vdots \\
\liminf _{x \rightarrow x_{0}} H_{n}(x)
\end{array}\right) \\
& \geq\left(\begin{array}{c}
H_{1}\left(x_{0}\right) \\
\vdots \\
H_{n}\left(x_{0}\right)
\end{array}\right)=H\left(x_{0}\right),
\end{aligned}
$$

that is,

$$
\liminf _{x \rightarrow x_{0}} \max _{w \in \Omega(x)} M(w) x \geq \max _{w \in \Omega\left(x_{0}\right)} M(w) x_{0} .
$$

If $\bar{x}$ satisfies (35), then

$$
\bar{x} \geq 0, \quad M(\bar{w}) \bar{x}>0, \quad \text { for some } \bar{w} \in \Omega(\bar{x})
$$

which is equivalent to

$$
\bar{x} \geq 0, \quad H(\bar{x})>0 .
$$

On the other hand, $\lim \inf _{\lambda \rightarrow 0^{+}} H(\bar{x}+\lambda e) \geq H(\bar{x})>0$, and $\bar{x}+\lambda e>0$ for $\lambda>0$. By taking $\lambda>0$ small enough, we know $\bar{x}+\lambda$ e satisfies (34). Thus, the proof is complete.

There is another point worthy of pointing out. We mentioned that the classical linear complementarity problem $\operatorname{LCP}(M, q)$ is feasible for all $q \in \mathbb{R}^{n}$ if and only if $M \in \mathbb{R}^{n \times n}$ is a $S$-matrix; that is, there exists $x \in \mathbb{R}^{n}$, such that

$$
x>0, \quad M x>0 .
$$

Is there any analogous result in the set-valued set? Yes, we have an answer for it in Theorem 5 below. 
Theorem 5. Consider the set-valued linear complementarity problem $\operatorname{SVLCP}(M, q, \Omega)$. If there exists $x \in \mathbb{R}^{n}$, such that

$$
x \geq 0, \quad M(w) x>0, \quad \text { for some } w \in \bigcap_{\widetilde{N} \in N_{\infty}} \bigcup_{n \in \widetilde{N}} \Omega(n x),
$$

then $\operatorname{SVLCP}(M, q, \Omega)$ is feasible for all $q: \mathbb{R}^{m} \rightarrow \mathbb{R}^{n}$ being bounded from below.

Proof. Let $q$ be any mapping from $\mathbb{R}^{m}$ to $\mathbb{R}^{n}$ being bounded from below, that is, there exists $\beta \in \mathbb{R}$, such that $q(w) \geq \beta e$. Suppose that $x_{0}$ and $w_{0}$ satisfy (45), which means

$$
x_{0} \geq 0, \quad M\left(w_{0}\right) x_{0}>0, \quad w_{0} \in \bigcap_{\widetilde{N} \in N_{\infty}} \bigcup_{n \in \widetilde{N}} \Omega\left(n x_{0}\right) .
$$

Then, for any $\widetilde{N} \in N_{\infty}$, we have $w_{0} \in \cup_{n \in \widetilde{N}} \Omega\left(n x_{0}\right)$. In particular, we observe the following:

(1) if taking $\widetilde{N}=\{1,2, \ldots$,$\} , then there exists n_{1}$, such that $w_{0} \in \Omega\left(n_{1} x_{0}\right)$;

(2) if taking $\widetilde{N}=\left\{n_{1}+1, \ldots,\right\}$, then there exists $n_{2}$ with $n_{2}>n_{1}$, such that $w_{0} \in \Omega\left(n_{2} x_{0}\right)$.

Repeating the above process yields a sequence $\left\{n_{k}\right\}$, such that $w_{0} \in \Omega\left(n_{k} x_{0}\right)$ and $n_{k} \rightarrow \infty$. Since $M\left(w_{0}\right) x_{0}>0$, it ensures the existence of $\alpha>0$, such that $M\left(w_{0}\right) x_{0}>\alpha e$. Taking $k$ large enough to satisfy $n_{k}>\max \{-\beta / \alpha, 0\}$ gives $\alpha n_{k} e>$ $-\beta e \geq-q(w)$. Then, it implies that

$$
M\left(w_{0}\right) n_{k} x_{0}>\alpha n_{k} e \geq-q(w),
$$

and hence

$$
n_{k} x_{0} \geq 0, \quad M\left(w_{0}\right)\left(n_{k} x_{0}\right)+q(w)>0, \quad w_{0} \in \Omega\left(n_{k} x_{0}\right),
$$

which says $n_{k} x_{0}$ is a feasible point of $\operatorname{SVLCP}(M, q, \Omega)$.

Definition 6. A matrix $M \in \mathbb{R}^{n \times n}$ is said to be a $P$-matrix if all its principal minors are positive, or equivalently [12, Theorem 3.3.4],

$\forall x \neq 0, \quad \exists k \in\{1,2, \ldots, n\}$ such that $x_{k}(M x)_{k}>0$.

From [12, Corollary 3.3.5], we know that every $P$-matrix is an $S$-matrix. In other words, if $M$ satisfies (49), then the following system is solvable:

$$
x \geq 0, \quad M x>0 .
$$

Their respective corresponding conditions in set-valued complementarity problem are

$$
\forall x \neq 0, \quad \exists k \in\{1, \ldots, n\} \quad \text { such that } x_{k}(M(w) x)_{k}>0,
$$
for some $w \in \Omega(x)$,

$$
x \geq 0, M(w) x>\quad \text { for some } w \in \Omega(x) .
$$

Example 7 shows that the aforementioned implication is not valid as well in set-valued complementarity problem.
Example 7. Let

$$
M(w)=\left(\begin{array}{cc}
w & 0 \\
0 & -w
\end{array}\right), \quad \Omega(x)= \begin{cases}-1, & x_{1}=0 \\
1, & \text { otherwise. }\end{cases}
$$

For $x_{1} \neq 0$, we have $M(w) x=\left(x_{1},-x_{2}\right)$, and hence $x_{1}(M(w) x)_{1}=x_{1}^{2}>0$. For $x_{1}=0$, we know $x_{2} \neq 0($ since $x \neq 0)$ which says $M(w) x=\left(-x_{1}, x_{2}\right)$, and hence $x_{2}(M(w) x)_{2}=$ $x_{2}^{2}>0$. Therefore, condition (51) is satisfied. But, condition (52) fails to hold because $M(w) x=\left(x_{1},-x_{2}\right)$ or $\left(-x_{1}, x_{2}\right)$. Hence, $M(w) x>0$ implies that $x_{2}<0$ or $x_{1}<0$, which contradicts with $x \geq 0$.

Definition 8. A matrix $M \in \mathbb{R}^{n \times n}$ is said to be semimonotone if

$$
\forall 0 \neq x \geq 0 \Longrightarrow \exists x_{k}>0 \quad \text { such that }(M x)_{k} \geq 0 .
$$

For the classical linear complementarity problem, we know that $M$ is semimonotone if and only if $\operatorname{LCP}(M, q)$ with $q>0$ has a unique solution (zero solution), see [12, Theorem 3.9.3]. One may wonder whether such fact still holds in set-valued case. Before answering it, we need to know how to generalize concept of semi-monotonicity to its corresponding definition in the set-valued case.

Definition 9. The set of matrices $\{M(w) \mid w \in \Omega(x)\}$ is said to be

(a) strongly semimonotone if for any nonzero $x \geq 0$,

$\exists x_{k}>0$ such that $(M(w) x)_{k} \geq 0 \quad \forall w \in \Omega(x)$;

(b) weakly semimonotone if for any nonzero $x \geq 0$,

$\exists x_{k}>0$ such that $(M(w) x)_{k} \geq 0$ for some $w \in \Omega(x)$.

Unlike the classical linear complementarity problem case, here are parallel results regarding set-valued linear complementarity problem which strong (weak) semi-monotonicity plays in.

Theorem 10. For the $\operatorname{SVLCP}(M, q, \Omega)$, the following statements hold.

(a) If the set of matrices $\{M(w) \mid w \in \Omega(x)\}$ is strongly semi-monotone, then for any positive mapping $q$, that is, $q(w)>0$ for all $w, \operatorname{SVLCP}(M, q, \Omega)$ has zero as its unique solution.

(b) If $\operatorname{SVLCP}(M, q, \Omega)$ with $q(w)>0$ has zero as its unique solution, then the set of matrices $\{M(w) \mid w \in \Omega(x)\}$ is weakly semi-monotone.

Proof. (a) It is clear that, for any positive mapping $q, x=0$ is a solution of $\operatorname{SVLCP}(M, q, \Omega)$. Suppose there is another nonzero solution $\bar{x}$, that is, $\exists \bar{w} \in \Omega(\bar{x})$, such that

$$
\bar{x} \geq 0, \quad M(\bar{w}) \bar{x}+q(\bar{w}) \geq 0, \quad \bar{x}^{T}(M(\bar{x})+q(\bar{w}))=0 .
$$


It follows from (55) that there exists $k \in\{1,2, \ldots, n\}$, such that $\bar{x}_{k}>0$ and $(M(\bar{w}) \bar{x})_{k} \geq 0$, and hence $(M(\bar{w}) \bar{x}+q(\bar{w}))_{k}>0$, which contradicts condition (57).

(b) Suppose $\{M(w) \mid w \in \Omega(x)\}$ is not weakly semi-monotone. Then, there exists a nonzero $\bar{x} \geq 0$, for all $k \in I^{+}(\bar{x}):=$ $\left\{i \mid \bar{x}_{i}>0\right\},(M(w) \bar{x})_{k}<0$ for all $w \in \Omega(\bar{x})$. Choose $\bar{w} \in$ $\Omega(\bar{x})$. Let $q(w)=1$ for all $w \neq \bar{w}$ and

$$
q_{k}(\bar{w})= \begin{cases}-(M(\bar{w}) \bar{x})_{k}, & k \in I^{+}(\bar{x}) \\ \max \left\{(M(\bar{w}) \bar{x})_{k}, 0\right\}+1, & \text { otherwise }\end{cases}
$$

Therefore, $q(w)>0$ for all $w$. According to the above construction, we have

$$
\begin{array}{r}
\bar{x} \geq 0, \quad M(\bar{w}) \bar{x}+q(\bar{w}) \geq 0, \quad \bar{x}^{T}(M(\bar{w}) \bar{x}+q(\bar{w}))=0, \\
\text { with } \bar{w} \in \Omega(\bar{x}),
\end{array}
$$

that is, the nonzero vector $\bar{x}$ is a solution of $\operatorname{SVLCP}(M, q, \Omega)$, which is a contradiction.

Theorem 10(b) says that the weak semi-monotonicity is a necessary condition for zero being the unique solution of $\operatorname{SVLCP}(M, q, \Omega)$. However, it is not the sufficient condition, see Example 11.

Example 11. Let

$$
M(w)=\left(\begin{array}{ccc}
-w & 1 & 0 \\
0 & 0 & 1 \\
1 & 0 & 0
\end{array}\right), \quad \Omega(x)=\{0,1\} .
$$

For any nonzero $x=\left(x_{1}, x_{2}, x_{3}\right) \geq 0$, we have $M(0) x=\left(x_{2}\right.$, $\left.x_{3}, x_{1}\right) \geq 0$. If we plug in $q=(1,1,1)$, by a simple cal-culation, $x=(1,0,0)$ satisfies

$$
x \geq 0, \quad M(1) x+q \geq 0, \quad x^{T}(M(1)+q)=0
$$

which means that $\operatorname{SVLCP}(M, q, \Omega)$ has a nonzero solution. We also notice that the set-valued mapping $\Omega(x)$ is even continuous in Example 11.

So far, we have seen some major difference between the classical complementarity problem and set-valued complementarity problem. Such phenomenon undoubtedly confirms that it is an interesting, important, and challenging task to study the set-valued complementarity problem, which, to some extent, is the main motivation of this paper.

To close this section, we introduce some other concepts which will be used later too. A function $f: \mathbb{R}^{n} \rightarrow \mathbb{R}$ is level bounded, if the level set $\{x \mid f(x) \leq \alpha\}$ is bounded for all $\alpha \in$ $\mathbb{R}$. The metric projection of $x$ to a closed convex subset $A \subset$ $\mathbb{R}^{n}$ is denoted by $\Pi_{A}(x)$, that is, $\Pi_{A}(x):=\arg \min _{y \in A}\|x-y\|$. The distance function is defined as $\operatorname{dist}(x, A):=\left\|x-\Pi_{A}(x)\right\|$.

\section{Properties of Solution Sets}

Recently, many authors study other classes of complementarity problems, in which another type of vector $w \in \Omega$ is involved, for example, the stochastic complementarity problem [14-17], to find $x \in \mathbb{R}^{n}$, such that

$$
x \geq 0, \quad F(x, w) \geq 0, \quad x^{T} F(x, w)=0, \quad \text { a.e. } \quad w \in \Omega,
$$

where $w$ is a random vector in a given probability space and the semi-infinite complementarity problem [18] to find $x \in \mathbb{R}^{n}$, such that

$$
x \geq 0, \quad F(x, w) \geq 0, \quad x^{T} F(x, w)=0, \quad \forall w \in \Omega,
$$

which we denote it by $\operatorname{SINCP}(F, \Omega)$. In addition, the authors introduce the following two complementarity problems in [18] to find $x \in \mathbb{R}^{n}$, such that

$$
\begin{aligned}
& x \geq 0, \quad F_{\text {min }}(x) \geq 0, \quad x^{T} F_{\text {min }}(x)=0, \\
& x \geq 0, \quad F_{\text {max }}(x) \geq 0, \quad x^{T} F_{\text {max }}(x)=0,
\end{aligned}
$$

where

$$
\begin{gathered}
F_{\min }(x):=\left(\begin{array}{c}
\min _{w \in \Omega} F_{1}(x, w) \\
\vdots \\
\min _{w \in \Omega} F_{n}(x, w)
\end{array}\right), \\
F_{\max }(x):=\left(\begin{array}{c}
\max _{w \in \Omega} F_{1}(x, w) \\
\vdots \\
\max _{w \in \Omega} F_{n}(x, w)
\end{array}\right) .
\end{gathered}
$$

These two problems are denoted by $\operatorname{NCP}\left(F_{\text {min }}\right)$ and $\operatorname{NCP}\left(F_{\max }\right)$, respectively. Is there any relationship among their solutions sets? In order to further describing such relationship, we adapt the following notations:

(i) $\operatorname{SOL}(F, \Omega)$ means the solution set of $\operatorname{SVNCP}(F, \Omega)$,

(ii) $\operatorname{SOL}(M, q, \Omega)$ means the solution set of $\operatorname{SVLCP}(F, \Omega)$,

(iii) $\widehat{\operatorname{SOL}}(F, \Omega)$ means the solution set of $\operatorname{SINCP}(F, \Omega)$,

(iv) $\operatorname{SOL}\left(F_{\text {min }}\right)$ means the solution set of $\operatorname{NCP}\left(F_{\text {min }}\right)$,

(v) $\operatorname{SOL}\left(F_{\max }\right)$ means the solution set of $\operatorname{NCP}\left(F_{\max }\right)$.

Besides, for the purpose of comparison, we restrict that $\Omega(x)$ is fixed; that is, there exists a subset $\Omega$ in $\mathbb{R}^{m}$, such that $\Omega(x)=$ $\Omega$ for all $x \in \mathbb{R}^{n}$.

It is easy to see that the solution set of $\operatorname{SINCP}(F, \Omega)$ is $\bigcap_{w \in \Omega} \operatorname{SOL}\left(F_{w}\right)$, but that of $\operatorname{SVNCP}(f, \Omega)$ is $\bigcup_{w \in \Omega} \operatorname{SOL}\left(F_{w}\right)$, where $F_{w}(x):=F(x, w)$. Hence, the solution set of $\operatorname{SINCP}(F, \Omega)$ is included in that of $\operatorname{SVNCP}(F, \Omega)$. In other words, we have

$$
\widehat{\operatorname{SOL}}(F, \Omega) \subseteq \operatorname{SOL}(F, \Omega)
$$

The inclusion (67) can be strict as shown in Example 12.

Example 12. Let $F(x, w)=(w, 1)$ and $\Omega(x)=[0,1]$. Then, we can verify that $\widehat{\operatorname{SOL}}(F, \Omega)=\{0,0\}$, whereas $\operatorname{SOL}(F, \Omega)=\{x \mid$ $\left.x_{1} \geq 0, x_{2}=0\right\}$. 
However, the solution set of $\operatorname{SVNCP}(F, \Omega), \operatorname{NCP}\left(F_{\text {min }}\right)$, and $\operatorname{NCP}\left(F_{\max }\right)$ are not included each other. This is illustrated in Examples 13-14.

Example 13. $\operatorname{SOL}\left(F_{\text {min }}\right) \nsubseteq \operatorname{SOL}(F, \Omega)$ and $\operatorname{SOL}(F, \Omega) \nsubseteq$ $\operatorname{SOL}\left(F_{\text {min }}\right)$.

(a) Let $F(x, w)=(1-w, w)$ and $\Omega=[0,1]$. Then, $\operatorname{SOL}\left(F_{\text {min }}\right)=\mathbb{R}_{+}^{2}, \operatorname{SOL}(F, \Omega)=\bigcup_{w \in \Omega} \operatorname{SOL}\left(F_{w}\right)=$ $\left\{\left(x_{1}, x_{2}\right)^{T} \mid x_{1} \geq 0, x_{2} \geq 0\right.$, and $\left.x_{1} x_{2}=0\right\}$.

(b) Let $F(x, w)=\left(w-1, x_{2}\right)$ and $\Omega=[0,1]$. Then, $\operatorname{SOL}\left(F_{\min }\right)=\emptyset$ and $\operatorname{SOL}(F, \Omega)=\left\{\left(x_{1}, x_{2}\right) \mid x_{1} \geq 0\right.$, $\left.x_{2}=0\right\}$.

Example 14. $\operatorname{SOL}\left(F_{\max }\right) \nsubseteq \operatorname{SOL}(F, \Omega)$ and $\operatorname{SOL}(F, \Omega) \nsubseteq$ $\operatorname{SOL}\left(F_{\max }\right)$.

(a) Let $F(x, w)=(w-1,-w)$ and $\Omega=[0,1]$. Then, $\operatorname{SOL}\left(F_{\max }\right)=\mathbb{R}_{+}^{2}$ and $\operatorname{SOL}(F, \Omega)=\emptyset$.

(b) Let $F(x, w)=(w,-w)$ and $\Omega=[0,1]$. Then, $\operatorname{SOL}\left(F_{\max }\right)=$ $\left\{\left(x_{1}, x_{2}\right) \mid x_{1}=0, x_{2} \geq 0\right\}$ and $\operatorname{SOL}(F, \Omega)=\mathbb{R}_{+}^{2}$.

Similarly, Example 15 shows that the solution set of NCP $\left(F_{\max }\right)$ and $\operatorname{NCP}\left(F_{\min }\right)$ cannot be included each other.

Example 15. $\operatorname{SOL}\left(F_{\max }\right) \nsubseteq \operatorname{SOL}\left(F_{\min }\right)$ and $\operatorname{SOL}\left(F_{\min }\right) \nsubseteq$ $\operatorname{SOL}\left(F_{\max }\right)$.

(a) Let $F(x, w)=(w-1,0)$ and $\Omega=[0,1]$. Then, $\operatorname{SOL}\left(F_{\min }\right)=\emptyset$ and $\operatorname{SOL}\left(F_{\max }\right)=\mathbb{R}_{+}^{2}$.

(b) Let $F(x, w)=(w, w)$ and $\Omega=[0,1]$. Then, $\operatorname{SOL}\left(F_{\min }\right)=$ $\mathbb{R}_{+}^{2}$ and $\operatorname{SOL}\left(F_{\max }\right)=\{(0,0)\}$.

In spite of these, we obtain some results which describe the relationship among them.

Theorem 16. Let $\Omega(x)=\Omega$ for all $x \in \mathbb{R}^{n}$. Then, we have

(a) $\operatorname{SOL}(F, \Omega) \cap\left\{x \mid F_{\min }(x) \geq 0\right\} \subseteq \operatorname{SOL}\left(F_{\min }\right)$;

(b) $\operatorname{SOL}\left(F_{\max }\right) \cap\{x \mid F(x, w) \geq 0$ for some $w \in \Omega\} \subseteq$ $\operatorname{SOL}(F, \Omega)$;

(c) $\operatorname{SOL}\left(F_{\text {min }}\right) \cap\left\{x \mid x^{T} F_{\text {max }}(x) \leq 0\right\}=\operatorname{SOL}\left(F_{\text {max }}\right) \cap\{x \mid$ $\left.F_{\min }(x) \geq 0\right\} \subseteq S O L(F, \Omega)$.

Proof. Parts (a) and (b) follow immediately from the fact

$$
x^{T} F_{\min }(x) \leq x^{T} F(x, w) \leq x^{T} F_{\max }(x) \quad \forall w \in \Omega, x \in \mathbb{R}_{+}^{n} .
$$

Part (c) is from (67), since the two sets in the left side of (c) is $\widehat{\operatorname{SOL}}(F, \Omega)$ by $[18]$.

For further characterizing the solution sets, we recall that for a set-valued mapping $M: \mathbb{R}^{n} \rightrightarrows \mathbb{R}^{m}$, its inverse mapping (see $[9$, Chapter 5]) is defined as

$$
M^{-1}(y):=\{x \mid y \in M(x)\} .
$$

Theorem 17. For $\operatorname{SVNCP}(F, \Omega)$, we have

$$
\operatorname{SOL}(F, \Omega)=\bigcup_{w \in \mathbb{R}^{m}}\left[\operatorname{SOL}\left(F_{w}\right) \cap \Omega^{-1}(w)\right] .
$$

Proof. In fact, the desired result follows from

$$
\begin{aligned}
& \operatorname{SOL}(F, \Omega) \\
&=\left\{x \mid x \in \operatorname{SOL}\left(F_{w}\right) \text { and } w \in \Omega(x)\right. \\
&\text { for some } \left.w \in \mathbb{R}^{m}\right\} \\
&=\left\{x \mid x \in \operatorname{SOL}\left(F_{w}\right) \text { and } x \in \Omega^{-1}(w)\right. \\
&\text { for some } \left.w \in \mathbb{R}^{m}\right\} \\
&= \bigcup_{w \in \mathbb{R}^{m}}\left[\operatorname{SOL}\left(F_{w}\right) \cap \Omega^{-1}(w)\right],
\end{aligned}
$$

where the second equality is due to the definition of inverse mapping given as above.

\section{Merit Functions for SVNCP and SVLCP}

It is well known that one of the important approaches for solving the complementarity problems is to transfer it to a system of equations or an unconstrained optimization via NCP functions or merit functions. Hence, we turn our attention in this section to address merit functions for $\operatorname{SVNCP}(F, \Omega)$ and $\operatorname{SVLCP}(M, q, \Omega)$.

A function $\phi: \mathbb{R}^{2} \rightarrow \mathbb{R}$ is called an NCP function if it satisfies

$$
\phi(a, b)=0 \Longleftrightarrow a \geq 0, \quad b \geq 0, a b=0 .
$$

For example, the natural residual $\phi_{\mathrm{NR}}(a, b)=\min \{a, b\}$ and the Fischer-Burmeister function $\phi_{\mathrm{FB}}(a, b)=\sqrt{a^{2}+b^{2}}-(a+b)$ are popular NCP-functions. Please also refer to [19] for a detailed survey on the existing NCP-functions. In addition, a real-valued function $f: \mathbb{R}^{n} \rightarrow \mathbb{R}$ is called a merit (or residual) function for a complementarity problem if $f(x) \geq 0$ for all $x \in \mathbb{R}^{n}$ and $f(x)=0$ if and only if $x$ is a solution of the complementarity problem. Given an NCP-function $\phi$, we define

$$
\begin{gathered}
r(x, w):=\|\Phi(x, F(x, w))\| \\
\text { where } \Phi(x, y):=\left(\phi\left(x_{1}, y_{1}\right), \ldots, \phi\left(x_{n}, y_{n}\right)\right)
\end{gathered}
$$

Then, it is not hard to verify that the function given by

$$
r(x):=\min _{w \in \Omega(x)} r(x, w)
$$

is a merit function for $\operatorname{SVNCP}(F, \Omega)$. Note that the merit function (74) is rather different from the traditional one, because the index set is not a fixed set, but dependent on $x$. We say that a merit function $r(x)$ has a global error bound with a modulus $c>0$ if

$$
\operatorname{dist}(x, \operatorname{SOL}(F, \Omega)) \leq c \cdot r(x), \quad \forall x \in \mathbb{R}^{n} .
$$

For more information about the error bound, please see [20] which is an excellent survey paper regarding the issue of error bounds. 
Theorem 18. Assume that there exists a set $\Omega \subset \mathbb{R}^{m}$, such that $\Omega(x)=\Omega$ for all $x \in \mathbb{R}^{n}$, and that for each $w \in \Omega, r(x, w)$ is a global error bound of $\operatorname{NCP}\left(F_{w}\right)$ with the modulus $\eta(w)>0$, that is,

$$
\operatorname{dist}\left(x, \operatorname{SOL}\left(F_{w}\right)\right) \leq \eta(w) r(x, w), \quad \forall x \in \mathbb{R}^{n} .
$$

In addition, if

$$
\eta:=\max _{w \in \Omega} \eta(w)<+\infty
$$

then $r(x)=\min _{w \in \Omega} r(x, w)$ provides a global error bound for $\operatorname{SVNCP}(F, \Omega)$ with the modulus $\eta$.

Proof. Noticing that if $\Omega(x)=\Omega$ for all $x \in \mathbb{R}^{n}$, then

$$
\Omega^{-1}(w)= \begin{cases}\mathbb{R}^{n}, & w \in \Omega, \\ \emptyset, & w \notin \Omega .\end{cases}
$$

It then follows from Theorem 17 that

$$
\operatorname{SOL}(F, \Omega)=\bigcup_{w \in \mathbb{R}^{m}}\left[\operatorname{SOL}\left(F_{w}\right) \cap \Omega^{-1}(w)\right]=\bigcup_{w \in \Omega} \operatorname{SOL}\left(F_{w}\right) .
$$

Therefore,

$$
\begin{aligned}
\operatorname{dist} & (x, \operatorname{SOL}(F, \Omega)) \\
& =\operatorname{dist}\left(x, \bigcup_{w \in \Omega} \operatorname{SOL}\left(F_{w}\right)\right) \\
& \leq \min _{w \in \Omega} \operatorname{dist}\left(x, \operatorname{SOL}\left(F_{w}\right)\right) \\
& \leq \min _{w \in \Omega} \eta(w) \cdot r(x, w) \\
& \leq \min _{w \in \Omega} \max _{w \in \Omega} \eta(w) \cdot r(x, w) \\
& =\max _{w \in \Omega} \eta(w) \min _{w \in \Omega} r(x, w) \\
& =\eta \cdot r(x) .
\end{aligned}
$$

Thus, the proof is complete.

One may ask when condition (77) is satisfied? Indeed, the condition (77) is satisfied if

(i) $\Omega$ is a finite set;

(ii) $F(x, w)=M(w) x+q(w)$ where $M(w)$ is continuous, and for each $w \in \Omega$ the matrix $M(w)$ is a $P$-matrix. In this case the modulus $\eta(w)$ takes an explicitly formula, that is,

$$
\eta(w)=\max _{d \in[0,1]^{n}}\left\|(I-D+D M(w))^{-1}\right\|
$$

see $[21,22]$. Hence, we see that

$$
\eta=\max _{\substack{d \in[0,1]^{n} \\ w \in \Omega}}\left\|(I-D+D M(w))^{-1}\right\|
$$

is well defined because $M(w)$ is continuous, and $\Omega$ is compact.

For simplification of notations, we write $x \rightarrow \infty$ instead of $\|x\| \rightarrow \infty$. We now introduce the following definitions which are similar to (29):

$$
\begin{aligned}
& \lim _{x \rightarrow \infty} \sup M(x) \\
& \quad:=\left\{u \mid \exists x^{n} \longrightarrow \infty, \exists u^{n} \longrightarrow u \text { with } u^{n} \in M\left(x^{n}\right)\right\}, \\
& \liminf _{x \rightarrow \infty} M(x) \\
& \quad:=\left\{u \forall x^{n} \longrightarrow \infty, \exists u^{n} \longrightarrow u \text { with } u^{n} \in M\left(x^{n}\right)\right\} .
\end{aligned}
$$

Definition 19. For $\operatorname{SVLCP}(M, q, \Omega)$, the set of matrices $\{M(w) \mid w \in \Omega(x)\}$ is said to have the limit- $R_{0}$ property if

$$
x \geq 0, \quad M(w) x \geq 0, \quad x^{T} M(w) x=0
$$$$
\text { for some } w \in \limsup _{x \rightarrow \infty} \Omega(x) \Longrightarrow x=0 \text {. }
$$

In the case of a linear complementarity problem, that is, $\Omega(x)$ is a fixed single-point set, Definition 19 coincides with that of $R_{0}$-matrix.

Theorem 20. For $\operatorname{SVLCP}(M, q, \Omega)$, suppose that there exists a bounded set $\Omega$, such that $\Omega(x) \subset \Omega$ for all $x \in \mathbb{R}^{n}$, and $M(w)$ and $q(w)$ are continuous on $\Omega$. If the set of matrices $\{M(w) \mid$ $w \in \Omega(x)\}$ has the limit- $R_{0}$ property, then the merit function $r(x)=\min _{w \in \Omega(x)}\|\min \{x, M(w) x+q(w)\}\|$ is level bounded.

Proof. We argue this result by contradiction. Suppose there exists a sequence $\left\{x_{n}\right\}$ satisfying $\left\|x_{n}\right\| \rightarrow \infty$, and $r\left(x_{n}\right)$ is bounded. Then,

$$
\begin{aligned}
\frac{r\left(x_{n}\right)}{\left\|x_{n}\right\|} & =\min _{w \in \Omega\left(x_{n}\right)}\left\|\min \left\{\frac{x_{n}}{\left\|x_{n}\right\|}, M(w) \frac{x_{n}}{\left\|x_{n}\right\|}+\frac{q(w)}{\left\|x_{n}\right\|}\right\}\right\| \\
& =\left\|\min \left\{\frac{x_{n}}{\left\|x_{n}\right\|}, M\left(w_{n}\right) \frac{x_{n}}{\left\|x_{n}\right\|}+\frac{q\left(w_{n}\right)}{\left\|x_{n}\right\|}\right\}\right\|,
\end{aligned}
$$

where we assume the minimizer is attained at $w_{n} \in \Omega\left(x_{n}\right)$, whose existence is ensured by the compactness of $\Omega\left(x_{n}\right)$, since $\Omega(x)$ is closed and $\Omega$ is bounded. Taking a subsequence if necessary, we can assume that $\left\{x_{n} /\left\|x_{n}\right\|\right\}$ and $\left\{w_{n}\right\}$ are both convergent in which $\bar{x}$ and $\bar{w}$ represent their corresponding limit point. Thus, we have

$$
\bar{w} \in \limsup _{n \rightarrow \infty} \Omega\left(x_{n}\right) \subset \lim _{\|x\| \rightarrow \infty} \sup \Omega(x) .
$$

Now, taking the limit in (85) yields

$$
\|\min \{\bar{x}, M(\bar{w}) \bar{x}\}\|=0,
$$

where we have used the fact that $q\left(w_{n}\right) /\left\|x_{n}\right\| \rightarrow 0$, because $q$ is continuous, and $w_{n} \in \Omega$ is bounded. This contradicts (84) since $\bar{x}$ is a nonzero vector. 
Note that the condition (84) is equivalent to

$$
\bigcup_{w \in \limsup _{x \rightarrow \infty} \Omega(x)} \operatorname{SOL}(M(w))=\{0\},
$$

which is also equivalent to saying that each matrix $M(w)$ for $w \in \lim \sup _{x \rightarrow \infty} \Omega(x)$ is a $R_{0}$-matrix.

Theorem 21. For $\operatorname{SVLCP}(M, q, \Omega)$, suppose that there exists a compact set $\Omega$, such that $\Omega(x) \subset \Omega$ for all $x \in$ $\mathbb{R}^{n}$, and $M(w)$ and $q(w)$ are continuous on $\Omega$. If $r(x)=$ $\min _{w \in \Omega(x)}\|\min \{x, M(w) x+q(w)\}\|$ is level bounded, then the following implication holds

$$
\begin{aligned}
& x \geq 0, \quad M(w) x \geq 0, \quad x^{T} M(w) x=0 \\
& \text { for some } w \in \bigcap_{\widetilde{N} \in N_{\infty}} \bigcup_{n \in \widetilde{N}} \Omega(n x) \Longrightarrow x=0 .
\end{aligned}
$$

Proof. Suppose that there exist a nonzero vector $x_{0}$, and $w_{0} \epsilon$ $\cap_{\widetilde{N} \in N_{\infty}} \cup_{n \in \widetilde{N}} \Omega\left(n x_{0}\right)$, such that

$$
x_{0} \geq 0, \quad M\left(w_{0}\right) x_{0} \geq 0, \quad x_{0}^{T} M\left(w_{0}\right) x_{0}=0 .
$$

Similar to the argument as in Theorem 5, there exists a sequence $\left\{n_{k}\right\}$ with $n_{k} \rightarrow \infty$ and $w_{0} \in \Omega\left(n_{k} x_{0}\right)$. Hence,

$$
\begin{aligned}
r\left(n_{k} x_{0}\right) & =\min _{w \in \Omega\left(n_{k} x_{0}\right)}\left\|\min \left\{n_{k} x_{0}, n_{k} M(w) x_{0}+q(w)\right\}\right\| \\
& \leq\left\|\min \left\{n_{k} x_{0}, n_{k} M\left(w_{0}\right) x_{0}+q\left(w_{0}\right)\right\}\right\| \\
& \leq \sum_{i=1}^{n}\left\|\min \left\{n_{k}\left(x_{0}\right)_{i}, n_{k}\left(M\left(w_{0}\right) x_{0}\right)_{i}+q\left(w_{0}\right)_{i}\right\}\right\| .
\end{aligned}
$$

Next, we proceed the arguments by discussing the following two cases.

Case 1. For $\left(x_{0}\right)_{i}>0$, we have $\left(M\left(w_{0}\right) x_{0}\right)_{i}=0$ from (90). Since $\max _{w \in \Omega} q(w)$ is finite due to the compactness of $\Omega$ and the continuity of $q(w), n_{k}\left(x_{0}\right)_{i}>\max _{w \in \Omega} q(w)$ for $k$ sufficiently large. Therefore, we obtain

$$
\left\|\min \left\{n_{k}\left(x_{0}\right)_{i}, n_{k}\left(M\left(w_{0}\right) x_{0}\right)_{i}+q_{i}\left(w_{0}\right)\right\}\right\|=\left\|q_{i}\left(w_{0}\right)\right\| .
$$

Case 2. For $\left(x_{0}\right)_{i}=0$, by a simple calculation, we have

$$
\begin{aligned}
\| \min & \left\{n_{k}\left(x_{0}\right)_{i}, n_{k}\left(M\left(w_{0}\right) x_{0}\right)_{i}+q_{i}\left(w_{0}\right)\right\} \| \\
& \times\left\{\begin{array}{cl}
=0, & \text { if } n_{k}\left(M\left(w_{0}\right) x_{0}\right)_{i}+q_{i}\left(w_{0}\right) \geq 0, \\
\leq\left\|q_{i}\left(w_{0}\right)\right\|, & \text { if } n_{k}\left(M\left(w_{0}\right) x_{0}\right)_{i}+q_{i}\left(w_{0}\right)<0,
\end{array}\right\},
\end{aligned}
$$

where the inequality in the latter case comes from the fact that $q_{i}\left(w_{0}\right) \leq n_{k}\left(M\left(w_{0}\right) x_{0}\right)_{i}+q_{i}\left(w_{0}\right)<0$. Thus,

$$
r\left(n_{k} x_{0}\right) \leq \sum_{n=1}^{n}\left\|q_{i}\left(w_{0}\right)\right\| .
$$

This contradicts the level boundedness of $r(x)$ since $n_{k} x_{0} \rightarrow$ $\infty$.
The above conclusion is equivalent to say that for each $w \in \cap_{\widetilde{N} \in N_{\infty}} \cup_{n \in \mathbb{N}} \Omega(n x)$, the matrix $M(w)$ is a $R_{0}$-matrix. Finally, let us discuss a special case where the set-valued mapping $\Omega(x)$ has an explicit form, for example, $\Omega(x)=\{w \mid$ $H(x, w)=0$ and $G(x, w) \geq 0\}$, where $H: \mathbb{R}^{n} \times \mathbb{R}^{m} \rightarrow \mathbb{R}^{l_{1}}$ and $G: \mathbb{R}^{n} \times \mathbb{R}^{m} \rightarrow \mathbb{R}^{l_{2}}$. Then, the solution set can be further characterized.

Theorem 22. If $\Omega(x):=\{w \mid G(x, w) \geq 0, H(x, w)=0\}$, then

$$
\begin{aligned}
\operatorname{SOL}(F, \Omega)=\bigcup_{w \in \mathbb{R}^{m}}\left\{x \mid\left(\begin{array}{c}
x \\
0 \\
\alpha
\end{array}\right) \in \operatorname{SOL}\left(\Theta_{w}\right)\right. \\
\text { with } \left.\alpha \in \mathbb{R}_{++}^{l_{2}} \text { and } 0 \in \mathbb{R}^{l_{1}}\right\},
\end{aligned}
$$

where $\Theta_{w}: \mathbb{R}^{n} \rightarrow \mathbb{R}^{n+l_{1}+l_{2}}$ is defined as $\Theta_{w}(x):=\left(\begin{array}{c}F(x, w) \\ G(x, w) \\ H(x, w)\end{array}\right)$ and $\mathbb{R}_{++}^{l_{2}}:=\left\{\alpha \in \mathbb{R}^{l_{2}} \mid \alpha_{i}>0\right.$ for all $\left.i=1, \ldots, l_{2}\right\}$.

Proof. Noting that the problem (2) is to find $w \in \mathbb{R}^{m}$ and $x \in \mathbb{R}^{n}$, such that

$$
\begin{gathered}
x \geq 0, \quad F(x, w) \geq 0, \quad\langle F(x, w), x\rangle=0, \\
G(x, w) \geq 0, \quad H(x, w)=0,
\end{gathered}
$$

namely, to find $w \in \mathbb{R}^{m}$ and $x \in \mathbb{R}^{n}$ satisfying

$$
\begin{gathered}
x \geq 0, \quad F(x, w) \geq 0, \quad\langle F(x, w), x\rangle=0, \\
0 \geq 0, \quad G(x, w) \geq 0, \quad 0 \cdot G(x, w)=0, \\
\alpha>0, \quad H(x, w) \geq 0, \quad\langle\alpha, H(x, w)\rangle=0 .
\end{gathered}
$$

In other words,

$$
\left(\begin{array}{l}
x \\
0 \\
\alpha
\end{array}\right) \geq 0, \quad\left(\begin{array}{l}
F(x, w) \\
G(x, w) \\
H(x, w)
\end{array}\right) \geq 0, \quad\left(\begin{array}{c}
x \\
0 \\
\alpha
\end{array}\right)^{T}\left(\begin{array}{c}
F(x, w) \\
G(x, w) \\
H(x, w)
\end{array}\right)=0 .
$$

Then, the desired result follows.

The foregoing result indicates that the set-valued complementarity problem is different from the classical complementarity problem, since it restricts that some components of the solution must be positive or zero, which is not required in the classical complementarity problems.

Moreover, the set-valued complementarity problem can be further reformulated to be an equation, that is, finding $x \in$ $\mathbb{R}^{n}$ and $w \in \mathbb{R}^{m}$ to satisfy the following equation

$$
\Gamma(x, w)=\left(\begin{array}{c}
\xi(x, w) \\
H(x, w) \\
\operatorname{dist}^{2}\left(G(x, w) \mid \mathbb{R}_{+}^{l_{2}}\right)
\end{array}\right)=0,
$$

where $\xi(x, w)=(1 / 2)\left\|\Phi_{\mathrm{FB}}(x, F(x, w))\right\|^{2}$. Note that when $A$ is a closed convex set, then $\theta(x):=\operatorname{dist}^{2}(x, A)$ is continuously 
differentiable, and $\nabla \theta(x)=2\left(x-\Pi_{A}(x)\right)$. This fact together with $\left\|\phi_{\mathrm{FB}}\right\|^{2}$ being continuously differentiable implies the following immediately.

Theorem 23. Suppose that $G$ and $H$ are continuously differentiable, and $\phi$ is the Fischer-Burmeister function, then $\Gamma$ is continuously differentiable and

$$
J T(x, w)=\left(\begin{array}{c}
J_{x} \xi(x, w) \\
J_{x} H(x, w) \\
2\left(G(x, w)-\prod_{\mathbb{R}_{+}^{l_{2}}}(G(x, w))\right)^{T} J_{x} G(x, w) \\
J_{w} \xi(x, w) \\
J_{w} H(x, w) \\
2\left(\begin{array}{c}
\left.G(x, w)-\prod_{\mathbb{R}_{+}^{l_{2}}}(G(x, w))\right)^{J} J_{w} G(x, w)
\end{array}\right),
\end{array}\right.
$$

where

$$
\begin{aligned}
J_{x} \xi(x, w)= & \Phi_{F B}(x, F(x, w))^{T} \\
& \times\left[\mathscr{D}_{a}(x, F(x, w))\right. \\
& \left.\quad+\mathscr{D}_{b}(x, F(x, w)) J_{x} F(x, F(x, w))\right], \\
J_{w} \xi(x, w)= & \Phi_{F B}(x, F(x, w))^{T} \mathscr{D}_{b}(x, F(x, w)) \\
& \times J_{w} F(x, w) .
\end{aligned}
$$

Here $\mathscr{D}_{a}(x, F(x, w))$ and $\mathscr{D}_{b}(x, F(x, w))$ mean the sets of $n \times n$ diagonal matrices diag $\left(a_{1}(x, F(x, w)), \ldots, a_{n}(x, F(x, w))\right)$ and $\operatorname{diag}\left(b_{1}(x, F(x, w)), \ldots, b_{n}(x, F(x, w))\right)$, respectively, and

$$
\begin{aligned}
& \left(a_{i}(x, F(x, w)), b_{i}(x, F(x, w))\right) \\
& \begin{cases}=\frac{\left(x_{i}, F_{i}(x, w)\right)}{\sqrt{x_{i}^{2}+F_{i}^{2}(x, w)}}-(1,1), & \text { if }\left(x_{i}, F_{i}(x, w)\right) \neq 0, \\
\in \bigcup_{\theta \in[0,2 \pi]}\{\cos \theta, \sin \theta\}-(1,1), & \text { if }\left(x_{i}, F_{i}(x, w)\right)=0 .\end{cases}
\end{aligned}
$$

\section{Further Discussions}

In this paper, we have paid much attention to the set-valued complementarity problems which posses rather different features from those of classical complementarity problems. As suggested by one referee, we here briefly discuss the relation between stochastic variational inequalities and the set-valued complementarity problems. Given $F: \mathbb{R}^{n} \times \Xi \rightarrow$ $\mathbb{R}, X_{\xi} \subset \mathbb{R}^{n}$ and $\Xi \subset \mathbb{R}^{l}$, a set representing future states of knowledge, the stochastic variational inequalities is to find $x \in X_{\xi}$, such that

$$
(y-x)^{T} F(x, \xi) \geq 0, \quad \forall y \in X_{\xi}, \xi \in \Xi .
$$

If $X_{\xi}=\mathbb{R}_{+}^{n}$, then the stochastic variational inequalities reduce to the stochastic complementarity problem as follows:

$$
x \geq 0, \quad F(x, \xi) \geq 0, \quad x^{T} F(x, \xi)=0, \quad \xi \in \Xi .
$$

The optimization problem corresponding to stochastic complementarity problem is

$$
\min _{x \in \mathbb{R}_{+}^{n}} \mathbb{E}\{\|\Phi(x, F(x, \xi))\|\} .
$$

When $\Xi$ is a discrete set, say $\Xi:=\left\{\xi_{1}, \xi_{2}, \ldots, \xi_{v}\right\}$, then

$$
\mathbb{E}\{\|\Phi(x, F(x, \xi))\|\}=\sum_{i=1}^{v} P\left(\xi_{i}\right)\left\|\Phi\left(x, F\left(x, \xi_{i}\right)\right)\right\|,
$$

where $P\left(\xi_{i}\right)$ is the probability of $\xi_{i}$. If the optimal value of (105) is zero, then it follows from (106) that (104) coincides with

$$
\begin{array}{r}
x \geq 0, \quad F\left(x, \xi_{i}\right) \geq 0, \quad x^{T} F\left(x, \xi_{i}\right)=0, \\
\forall \xi_{i} \in \Xi \text { satisfying } P\left(\xi_{i}\right)>0 .
\end{array}
$$

When $\Xi$ is a continuous set, then

$$
\mathbb{E}\{\|\Phi(x, F(x, \xi))\|\}=\int_{\Omega}\left\|\Phi\left(x, F\left(x, \xi_{i}\right)\right)\right\| P(x) d x,
$$

where $P(x)$ is the density function. In this case, (104) takes the form of

$$
x \geq 0, \quad F(x, \xi) \geq 0, \quad x^{T} F(x, \xi)=0, \quad \text { a.e. } \xi \in \Xi,
$$

or equivalently there exists a subset $\Xi_{0} \subset \Xi$ with $P\left(\Xi_{0}\right)=0$, such that

$$
x \geq 0, \quad F(x, \xi) \geq 0, \quad x^{T} F(x, \xi)=0, \quad \forall \xi \in \Xi \backslash \Xi_{0} .
$$

Hence the stochastic complementarity problem is, in certain extent, a semi-infinite complementarity problem (SICP).

Due to some major difference between set-valued complementarity problems and classical complementarity problems, there are still many interesting, important, and challenging questions for further investigation as below, to name a few.

(i) How to extend other important concepts used in classical linear complementarity problems to setvalued cases (like $P_{0}, P_{*}, Z, Q, Q_{0}, S, \bar{S}$, copositive, column sufficient matrix, etc.)?

(ii) How to propose an effective algorithm to solve (99)?

(iii) Can we provide some sufficient conditions to ensure the existence of solutions? One possible direction is to use fixed-point theory. In fact, the set-valued complementarity problem is to find $x \in \mathbb{R}^{n}$, such that

$$
x=\max \{0, x-F(x, w)\}=\Pi_{\mathbb{R}_{+}^{n}}(x-F(x, w))
$$

$$
\text { for some } w \in \Omega(x) \text {, }
$$


that is,

$$
x \in \Pi_{\mathbb{R}_{+}^{n}}(x-\widetilde{F}(x)),
$$

where $\widetilde{F}(x):=\bigcup_{w \in \Omega(x)} F(x, w)$. Note that (112) is a fixed point of a set-valued mapping $\Pi_{\mathbb{R}_{+}^{n}}(I-\widetilde{F})$, where $I$ denotes the identify mapping.

\section{Acknowledgments}

The authors would like to thank three referees for their carefully reading and suggestions which help to improve this paper. J. Zhou is supported by National Natural Science Foundation of China (11101248, 11271233), Shandong Province Natural Science Foundation (ZR2010AQ026, ZR2012AM016), and Young Teacher Support Program of Shandong University of Technology. J. S. Chen is supported by National Science Council of Taiwan. G. M. Lee is supported by the National Research Foundation of Korea (NRF) Grant funded by the Korea government (MEST) (no. 2012-0006236).

\section{References}

[1] F. Facchinei and J. S. Pang, Finite-Dimensional Variational Inequalities and Complementarity Problems, Volume I and II, Springer, New York, NY, USA, 2003.

[2] J. S. Pang and M. Fukushima, "Quasi-variational inequalities, generalized Nash equilibria, and multi-leader-follower games," Computational Management Science, vol. 2, no. 1, pp. 21-56, 2005.

[3] M. S. Gowda, "On the extended linear complementarity problem," Mathematical Programming B, vol. 72, no. 1, pp. 33-50, 1996.

[4] O. L. Mangasarian and J. S. Pang, "The extended linear complementarity problem," SIAM Journal on Matrix Analysis and Application, vol. 16, pp. 359-368, 1995.

[5] J. S. Pang, "The implicit complementarity problem," in Nonlinear Programming, O. L. Mangasarian, R. R. Meyer, and S. M. Robinson, Eds., Academic Press, New York, NY, USA, 4 edition, 1981.

[6] M. A. Noor, "Some developments in general variational inequalities," Applied Mathematics and Computation, vol. 152, no. 1, pp. 199-277, 2004.

[7] M. A. Noor, "Extended general variational inequalities," Applied Mathematics Letters, vol. 22, no. 2, pp. 182-185, 2009.

[8] E. Polak, Optimization: Algorithms and Consistent Approximation, Springer, New York, NY, USA, 1997.

[9] R. T. Rockafellar, R. J. Wets, and R. J., Variational Analysis, Springer, New York, NY, USA, 1998.

[10] R. T. Rockafellar, Convex Analysis, Princeton University Press, Princeton, NJ, USA, 1970.

[11] J. P. Aubin and H. Frankowska, Set-Valued Analysis, Birkhauser, Boston, Mass, USA, 1990.

[12] R. Cottle, J. S. Pang, and R. Stone, The Linear Complementarity Problem, Academic Press, New York, NY, USA, 1992.

[13] M. Fiedler and V. Pták, "Some generalizations of positive definiteness and monotonicity," Numerische Mathematik, vol. 9, no. 2, pp. 163-172, 1966.
[14] X. Chen and M. Fukushima, "Expected residual minimization method for stochastic linear complementarity problems," Mathematics of Operations Research, vol. 30, no. 4, pp. 1022-1038, 2005.

[15] X. Chen, C. Zhang, and M. Fukushima, "Robust solution of monotone stochastic linear complementarity problems," Mathematical Programming, vol. 117, no. 1-2, pp. 51-80, 2009.

[16] H. Fang, X. Chen, and M. Fukushima, "Stochastic $R_{0}$ matrix linear complementarity problems," SIAM Journal on Optimization, vol. 18, no. 2, pp. 482-506, 2007.

[17] C. Zhang and X. Chen, "Smoothing projected gradient method and its application to stochastic linear complementarity problems," SIAM Journal on Optimization, vol. 20, no. 2, pp. 627-649, 2009.

[18] J. C. Zhou, N. H. Xiu, and J. S. Chen, "Solution properties and error bounds for semi-infinite complementarity problems," Journal of Industrial and Management Optimization, vol. 9, pp. 99-115, 2013.

[19] A. Galantai, "Properties and construction of NCP functions," Computational Optimization and Applications, vol. 52, pp. 805824, 2012.

[20] J. S. Pang, "Error bounds in mathematical programming," Mathematical Programming B, vol. 79, no. 1-3, pp. 299-332, 1997.

[21] X. Chen and S. Xiang, "Computation of error bounds for $P$ matrix linear complementarity problems," Mathematical Programming, vol. 106, no. 3, pp. 513-525, 2006.

[22] S. A. Gabriel and J. J. More, "Smoothing of mixed complementarity problems," in Complementarityand Variational Problems, M. C. Ferris and J. S. Pang, Eds., SIAM Publications, Philadelphia, Pa, USA, 1997. 


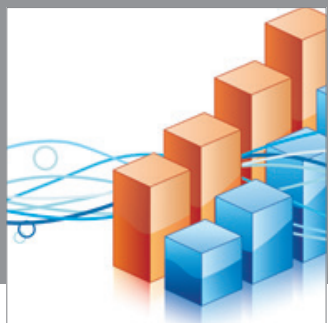

Advances in

Operations Research

mansans

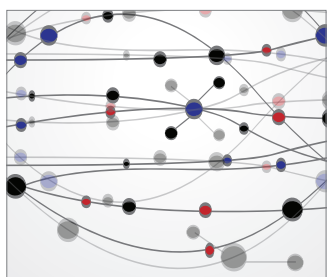

The Scientific World Journal
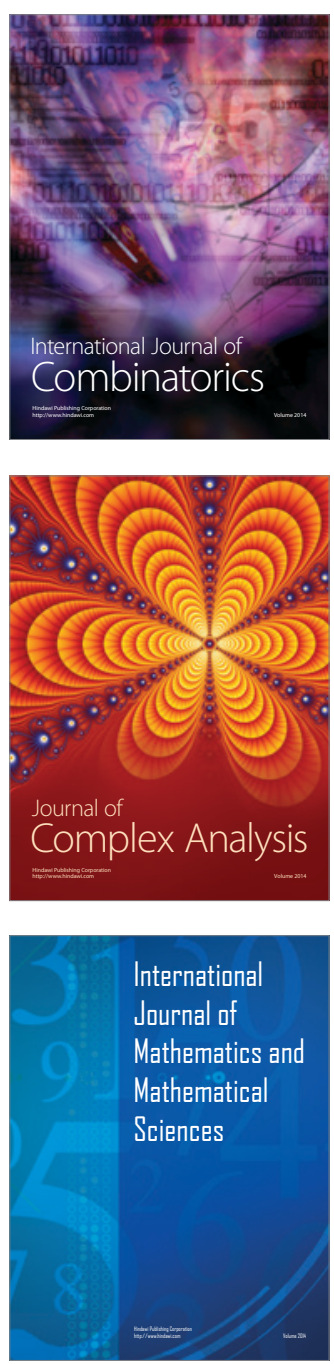
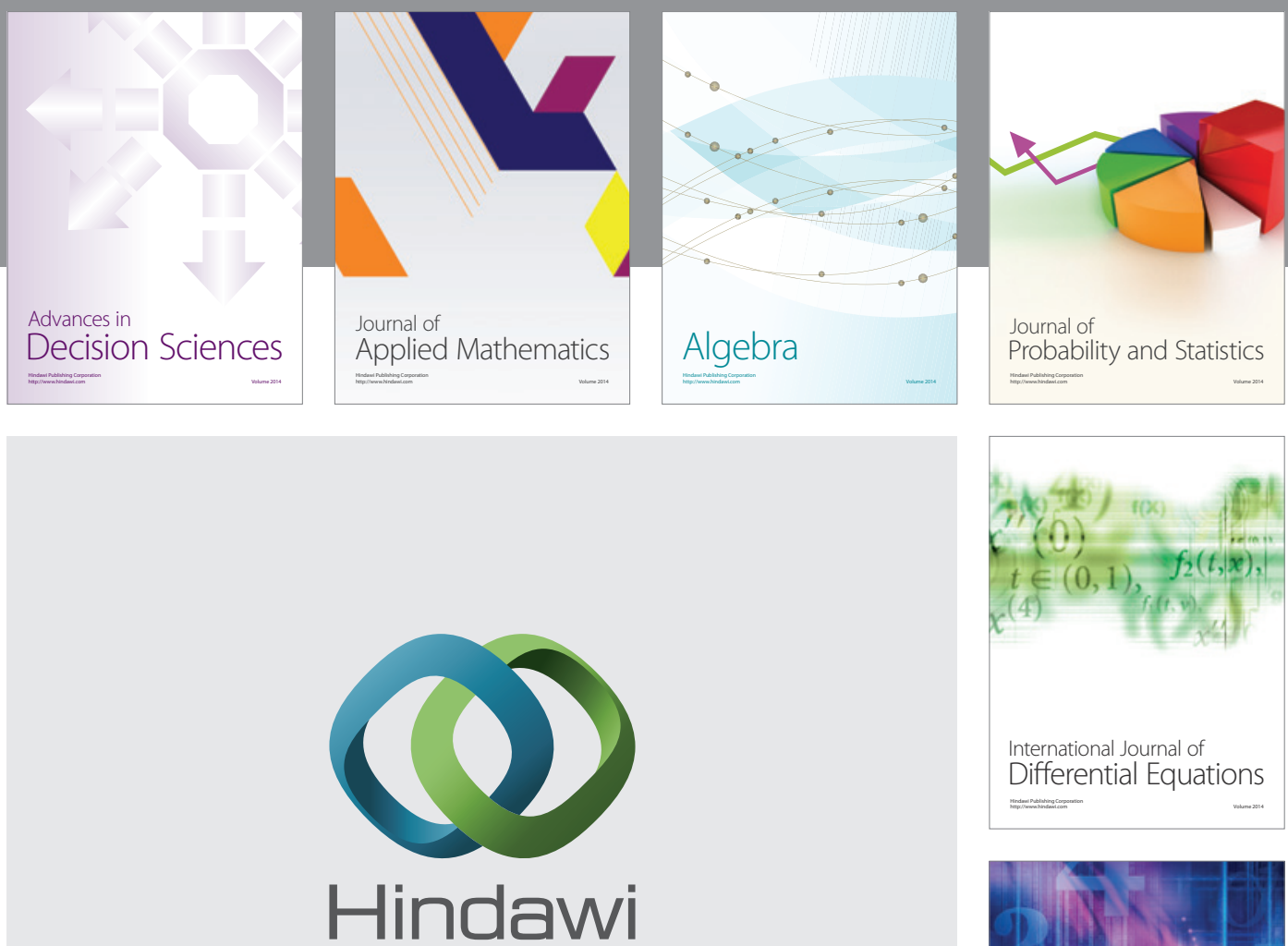

Submit your manuscripts at http://www.hindawi.com
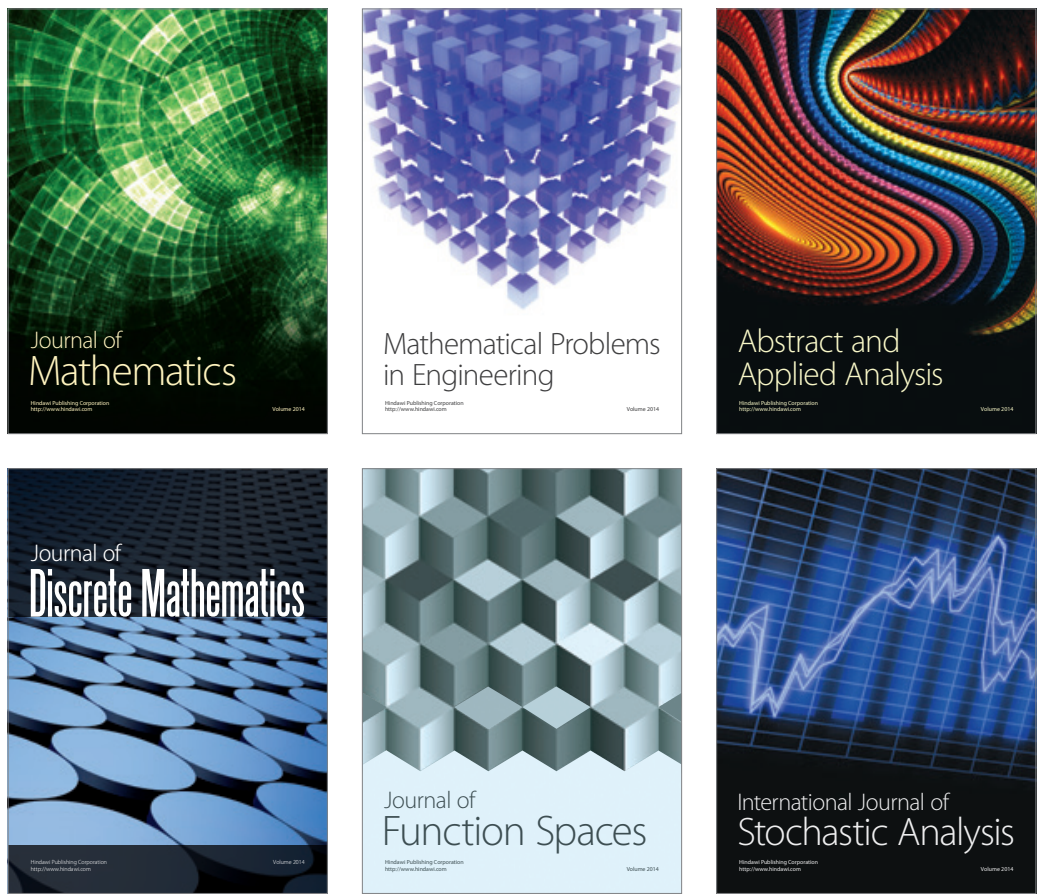

Journal of

Function Spaces

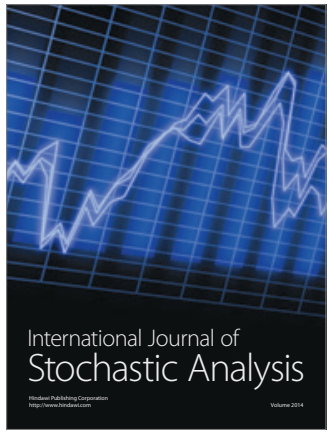


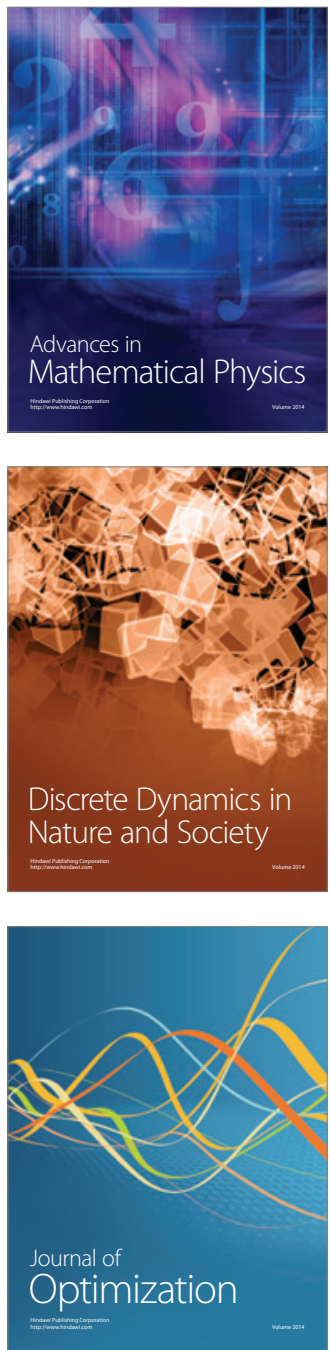